\title{
Teaching Intelligence System Based on the Cloud Platform of the Internet of Things and Its Application in Physical Education
}

\author{
Yuehua Chen, ${ }^{1}$ Bin Gao ${ }^{D},{ }^{2}$ and Huannan Cao $(\mathbb{D})^{3}$ \\ ${ }^{1}$ Physical Education and Research Office, Shijiazhuang Engineering \& Technology School, Shijiazhuang, 050061 Hebei, China \\ ${ }^{2}$ Department of Winter Sport, Hebei Sport University, Shijiazhuang, 050041 Hebei, China \\ ${ }^{3}$ Department of Social Sport, Hebei Sport University, Shijiazhuang, 050041 Hebei, China
}

Correspondence should be addressed to Huannan Cao; 2005009@hepec.edu.cn

Received 22 October 2021; Revised 18 November 2021; Accepted 26 November 2021; Published 20 January 2022

Academic Editor: Deepak Kumar Jain

Copyright (c) 2022 Yuehua Chen et al. This is an open access article distributed under the Creative Commons Attribution License, which permits unrestricted use, distribution, and reproduction in any medium, provided the original work is properly cited.

With the progress of physical education reform, more and more educational methods are added to physical education. The state has always attached great importance to the reform of school physical education and has promulgated and implemented a number of policies and documents to stimulate the reform of school physical education. Change the teaching method from "theoretical knowledge teaching" to "combination of theory and practice", change the teaching content from "subject standard and complicated subjects" to "comprehensive course teaching", and change "conservative and mechanical teaching" to "active and teacher-student participation". The introduction of intelligent systems and responsive software in physical education not only allows students to learn with the aid of multimedia courseware but also accesses to high-performance graphics data processing software GIMP (GNU Image Manipulation Program) and so on. This article describes the production plan of multimedia courseware under the intelligent system environment and constructs the sports teaching framework of multimedia courseware under the intelligent system desktop system. Choose the Ubuntu18.04 stable version system to obtain OpenOffice and GIMP components, as well as multimedia materials for physical education, and make physical education courseware. The experiment teaching effect evaluation is carried out in the main body composed of 100 physical education students in our college. Research shows that the use of GIMP can greatly improve the learning efficiency in the physical education of the intelligent system. Compared with Windows and other systems, the teaching system constructed in this study only retains the core components and can reflect great system permissions in teaching advantage. In terms of processing speed, the image processing time is reduced by $36 \%$ compared to Windows, and it has more types of professional materials than Microsoft. The learning time of teachers using the intelligent system professional physical education software is more than $50 \%$ shorter than that of Photoshop, 3D Max, etc., thereby improving the work efficiency of physical education teachers.

\section{Introduction}

With the rapid development of information technology, the Internet of Things, big data, and cloud computing have become the only way for traditional industries to upgrade, increase value-added services, and improve product competitiveness. Cloud platforms provide technologies for traditional industries to enter the Internet of Things field support. The massive data accumulated by massive equipment for a long time is gathered on the cloud platform to form big data. The use of cloud computing for big data analysis can extract poten- tial value information from the data and provide a data source for higher forms of artificial intelligence. Physical education teaching and research workers also gradually began to realize the importance of the effectiveness test of curriculum experimental intervention. The reason is that in the past, physical education teachers received the same training in the experiment, but the degree of implementing the curriculum model in teaching is different. In addition, in the field of physical education, the effectiveness of the experimental intervention is closely related to the experimental results because the intervention of each influencing factor may affect the experimental 
results. Using IoT cloud platform to make multimedia courseware, the definition of experimental intervention execution effectiveness is guided, which can be divided into health and pedagogy.

In the core grammar research field of the intelligent teaching system for the research object of this research, Quach et al. propose a fixed method, aiming at the error problems in large-scale programs, and propose to extract relevant code fragments based on syntax search and transform them to generate candidate titles [1]. Stuermer et al. proposed that sports researchers should use formal and reasonable test methods to comprehensively reflect the implementation quality of experimental intervention. They also suggested that environmental factors should be measured when measuring the effectiveness of experimental interventions [2]. Yuan et al. put forward the teacher's specialty, students' previous curriculum experience, teaching time, and other factors that affect the intervention. However, the measurement and evaluation of the effectiveness of experimental intervention are not unified, and there are many differences [3]. Block and Dewald propose code phase and search repair, which use semantic search to obtain code fragments related to errors and transform them to generate candidate titles [4]. Wang et al. propose the cap gen method to improve the quality of titles by using finer-grained materials than sentence-level and using wrong context similarity to select repair templates. Although automatic program repair and title recommendation are different in error location and title quality judgment, they both need to generate titles [5]. Coudron and Secci proposed that there is no unified conceptual framework to guide the measurement and evaluation of the effectiveness of experimental intervention in the field of physical education [6].

In addition, in the PEZ study, the authors López and Baydal used the observation list to test the effectiveness of intervention implementation for all the exercise tasks in the teaching plan. In this paper, we propose some teaching strategies for cluster computing course. The virtualizationbased platform is used to build the cluster prototype, and Linux is used as its operating system. The evaluation of the course shows that students' knowledge and skills of the subject have improved at the end of the course. [7]. The GenProg method proposed by Belyaev and Ray assumes that the code fragments to be repaired exist in the source code of the program to be repaired. A genetic algorithm is used to cross mutate these code fragments to generate candidate titles, and test sets are used to detect the correctness of the output titles [8]. Mishra and Somani proposed the PAR method, which assumes that the materials for repairing errors exist in the source code of other projects and generates candidate titles by manually defining repair templates and randomly selecting constrained mutation operation, which overcomes the shortage of generating meaningless candidate titles by random mutation based on genetic algorithm $[9,10]$. Belyaev and Ray think that the ability of different repair templates is not the same, so high-frequency repair templates are preferred to generate candidate titles, which improve the accuracy of title generation compared with the PAR method $[11,12]$. The application of the Inter- net of Things provides a good reference, helps promote the entry of traditional industries into the field of Internet of Things, realizes the service interconnection of different smart service providers, and plays a vital role in promoting the development and promotion of the Internet of Things industry. The above studies are all related to the specific content of the intelligent cloud platform, such as the processing and analysis of the coding data of intelligent teaching, the effect of intervention, and the recognition of sentences. These studies study the core of the intelligent platform and put it in the material. The connection and inquiry on the networked teaching platform need to be strengthened.

As the technical core of the Internet of Things system, the cloud platform must first provide a service interface for the access between the user and the device; secondly, it needs to provide a communication channel for instant communication between the cloud and the device, and the device needs to be able to communicate with different devices. The sensor data generated by the terminal is analyzed and stored. This paper describes the making scheme of multimedia courseware under an IoT cloud platform environment and constructs the PE teaching framework of multimedia courseware under the smart teaching desktop system. Using the stable version of the Ubuntu 18.04 system, we can obtain OpenOffice and GIMP components, as well as multimedia materials of physical education, and make physical education courseware. The experimental teaching effect was evaluated in the main body composed of 100 students majoring in physical education in our college.

\section{Experiment and Method}

2.1. Research Content. Use the Internet as a platform to transmit teaching content, realize online teaching, and be able to communicate and learn online. The construction of an online learning system platform can break the limitations of traditional classroom education and form an active, collaborative, and open teaching mode. It has the advantages of vivid image and extensive resources and can visit and communicate with each other which are excellent characteristics of time and space constraints. This paper describes the making scheme of multimedia courseware under a smart IoT cloud platform environment, designs and implements the machine learning model, and uses the collected data set to train and generate a classifier that can recognize the repair title of courseware. This paper implements a tool system that can continuously collect and label security courseware repair titles and bug repair titles, and gives its design and implementation details. The system can be extended to support other open-source software managed by GIT, SVN, and another version 3 computer research and development control tools. Do not blindly believe or over rely on NVD, open-source software community courseware notice, because its comprehensiveness and timeliness are insufficient.

2.2. Physical Education Arrangement. The students who participated in the sports test were ranked according to the results of the sports test. The odd students were divided into the experimental group, and the even students were divided 
into the control group (according to the ranking order, the odd number is the experimental combination and the even number is the control group, with 50 people in each group). The specific designs of the experiment are as follows: on the basis of the normal physical education teaching mode, the experimental group, in addition to once a week of physical education extracurricular, physical education teachers for sports students on 30 minutes of integration theory, such as sports biomechanics, sports physiology, sports injury, and other theoretical knowledge, sports students according to the theory of integration of sports and medicine to develop a personal sports training program, twice a week for training. The experiment lasted for 32 weeks. The main content of normal teaching mode is divided into preparation part (warm-up exercise before exercise and explanation of exercise content), basic part (study and application of theoretical knowledge of motor skills), and ending part (relaxation of body, summary, and arrangement of motor skills). The main contents of the theory course are as follows: to explain and solve the doubts about the sports-related knowledge involved and to play the video of the principle of body movement. The main contents of the dynamic training program are as follows: according to the content of theoretical learning, teachers carry out specific demonstrations to guide students to practice and consolidate independently.

This paper compiles a tool to count all intelligent teaching system kernel courseware included in NVD physical education multimedia. There are a total of 4064 Linux kernel courseware included and confirmed by NVD physical education multimedia. Then, the tool tracks NVD and system kernel source code warehouse and finds only 1633 system kernel courseware whose repair title has been registered in NVD tracking; that is, NVD has confirmed the included intelligent teaching system kernel courseware; among them, only $40.2 \%$ of the core courseware repair titles are registered and associated with the NVD physical education multimedia, so that the NVD physical education multimedia can be recognized reversely. The tool of this paper tracks the source code warehouse of intelligent teaching system kernel directly and finds that there are only 380 courseware repair titles in it.

\subsection{Intelligent Teaching System in Physical Education} Teaching of Linux Desktop System. With the increasing complexity of software systems, the increasing application of open-source software, the transparency, and efficiency of courseware disclosure are improved, and governments and enterprises pay more attention to software security and increase investment; the number of software courseware disclosure is increasing year by year. At the same time, the software is penetrating into all aspects of economic and social life. Computer teaching system kernel and basic library (such as OpenSSL) are widely shared by different software systems. The use of its security courseware often affects a wide range of software systems and a large number of teachers. Therefore, it is very important to help teachers to timely apply the title repair of safety courseware in physical education. The life cycle of open-source software courseware can be divided into $k$ stages. The formula can be expressed as follows:

$$
\begin{gathered}
k_{t 1}[i]=\sum_{j} \log \left(\frac{\left|y_{A_{i}}-y_{A_{j}}\right|}{h_{A_{j}}}\right), \log \left(\frac{w_{A_{i}}}{w_{A_{j}}}\right)\left(w_{i}^{1}, w_{j}^{2}\right), \quad(1) \\
\eta N \lambda\left(A_{i}, A_{j}\right)=\left[\log \left(\frac{\left|x_{A_{i}}-a_{A_{j}}\right|}{w_{A_{j}}}\right), \log \left(\frac{\left|y_{A_{i}}-y_{A_{j}}\right|}{h_{A_{j}}}\right), \log \left(\frac{w_{A_{i}}}{w_{A_{j}}}\right), \log \left(\frac{h_{A_{i}}}{h_{A_{j}}}\right)\right] .
\end{gathered}
$$

Teachers use the courseware to repair the title to the software system. In the third stage of the software courseware life cycle, it is easy to be ignored and there is a lack of security prompt information, which leads to an obvious lack of management in the fourth stage, which affects the timely application of security title and brings security threats to teachers. This stage has attracted extensive attention of scholars, such as courseware detection, courseware mining, courseware analysis, and courseware utilization, which have been a research hotspot in the field of security and have been closely concerned by scientific research, enterprises, government agencies, and so on. Open-source software maintainers often quietly repair the security courseware; that is, it is very common that the code part of the courseware repair title and the commit message do not give the security description and ID information. For example, according to the research report statistics, when the courseware repair title is released, only $a$ has the ID clearly marked in the file by the maintainer, and only the maintainer of $u$ and $W$ has informed the security monitoring service provider that the courseware has been repaired in the release instructions of the new version.

$$
\begin{gathered}
\mathrm{ID}_{i j}=\frac{A^{b_{i j}}}{\sum_{k} A^{b_{i k}}}, \\
A_{(j \mid i)}=w_{i j} U_{i}, \\
U(k)=\frac{\|w\|^{2}}{1+\|w\|^{2}} \frac{w}{\|w\|} .
\end{gathered}
$$

However, compared with the release of the security title, the release of the new version of the software lags behind a lot and many basic services of the production environment; for security and stability considerations, administrators prefer to upgrade the title rather than upgrade the entire software version; administrators have to identify the specific security repair title. In this stage, the reaction is fast and easy to get priority treatment. Once the new security courseware is found and confirmed, the maintainer of the open-source community will actively respond and quickly develop, review, and process the repair title of the courseware. For example, according to the statistics in this paper, the repair reaction speed of the security courseware is $h$ times faster than that of the performance bug.

$$
H_{t}=\tanh \left(w_{c} x_{t}+u_{c}\left(r_{t} \Theta B_{t-1}\right)+B_{c}\right),
$$




$$
\frac{B_{t}}{H}=z_{t} \Theta h_{t-1}+\left(1-z_{t}\right) \Theta h_{t} .
$$

The number of developers allocated for security courseware is $Z$ times of that of performance bugs and $R$ times of that of other bugs. Through the comment of the title, especially the commit message of the title, it is clear that the title is to repair the security courseware, and the corresponding CVE ID (common vulnerabilities and exposure identifiers) is given. The teachers of the software (security software engineer, operating system release, software integrator, operation and maintenance engineer, etc.) are able to identify the repair title of the security courseware. It is very important to apply it to the software system as soon as possible.

$$
\begin{gathered}
W\left(\frac{H}{B}\right)=\frac{U+A}{U+A+H+B}, \\
L_{t 1}=A D * \operatorname{Test}\left(k_{t 1}\right), \\
A D=\frac{A /(A+H)}{w_{c} x_{t}+u_{c}\left(r_{t} \Theta B_{t-1}\right)} .
\end{gathered}
$$

Therefore, the proportion of intelligent teaching system kernel maintainers clearly marking CVE ID in the security repair title file is very low, just like the overall situation of open-source software. This leads teachers (even professional security engineers) to identify the security courseware repair title, so as to avoid missing and apply the security title as soon as possible. Based on these observations and analysis, an intelligent automatic recognition tool for the safe repair title is developed to realize the timely recognition and notification of the courseware repair title and reduce the workload and omission of manual recognition. Therefore, taking the representative Linux kernel source code community as an example, this paper designs and implements an automatic recognition method of Linux security courseware title. The core idea of this method is to define the courseware features for the code and description part of the title incorporated into the Linux kernel source code, construct the machine learning model $G$, and train the classifier $t$ that can recognize the safe courseware title $E$. The implementation of this method mainly includes the following steps:

$$
\begin{gathered}
T_{i k j l}= \begin{cases}\frac{G}{E_{i k j l}} \sqrt{\sum_{s=1}^{n}\left(x_{i k}(\varepsilon)-x_{j l}(\varepsilon)\right)^{2} E_{i k j l}(\varepsilon)}, & E_{i k j l}>0 \\
0, & E_{i k j l}<0\end{cases} \\
E_{t}=\sigma\left(w_{z} x_{t}+u_{z} T_{t-1}+G_{z}\right) .
\end{gathered}
$$

The experimental results show that the fine-grained classification of title types is effective but not significant, so it cannot reach the practical level of the research goal of this paper. This paper proposes a research method to identify courseware repair from bug repair titles. This method uses llvm to precompile the source code of the file modified by the title into IR and then uses the manually established title mode to pre- process the IR against the title mode and collect the pattern data.

2.4. Classification of Physical Education Courseware Based on Machine Learning. In recent years, the use of machine learning technology to analyze and process the program source code has become one of the research hotspots, in which the analysis of software courseware, bug repair, is an important research aspect. The following describes the most relevant work with this article, as well as the difference between this work and related work. This paper proposes a research method of automatically identifying the title of security courseware by using the log message and bug report extraction features of the title; the method in this paper extracts features from the log message and code of the title at the same time and combines the two aspects of feature information to build the model. Therefore, the feature engineering and modeling methods of modeling are obviously different. The complexities $O(L), O(c)$, and $O(b)$ of different features can be expressed as follows:

$$
\begin{gathered}
O(L)=p_{1} \mu_{1}+p_{2} \mu_{2}=\sum_{i=0}^{L-1} i p_{i}, \\
O(C)=p_{1}\left(\mu_{1}-\mu_{0}\right)^{2}+p_{2}\left(\mu_{2}-\mu_{0}\right)^{2}, \\
O(B)=\arg \max _{0 \leq t \leq L-1}\left\{p_{1}\left(\mu_{1}-\mu_{0}\right)^{2}+p_{2}\left(\mu_{2}-\mu_{0}\right)^{2}\right\},
\end{gathered}
$$

where $u$ is the coefficient of the cloud platform project.

This paper proposes a method to extract features from the log message and code parts of the title to identify the courseware title. Although both the log and code parts of the title are used in this paper, there are obvious differences between the feature engineering and modeling methods in this paper. For example, in this paper, the code is only used as pure text to extract keywords and then merge with the natural language part of the log. The method of this paper analyzes the code part according to the programming language and extracts its identifier, loop, code modification, and other fine-grained information to construct features, and this paper constructs the machine learning model by separating the features of code and log part, so the modeling method is also obviously different. IoT cloud platform intelligence physical education framework is shown in Figure 1.

In this paper, we propose to use the statistics (locality, complexity, and control loop) of the title combined with keywords to extract features and then use machine learning method to classify the security repair title more finely, such as buffer error (such as stack overflow), injection error (such as error injection), and numeric error (such as integer overflow) in this paper; the statistical characteristics of the title are used to model, but the research goal and the method of building the model are completely different. Finally, the collected data are provided as constraints to the manually defined symbol rules for constraint solving, and the result of solving is to judge whether it is a safe title [13]. This method provides a referential idea for the syntax information of the application, but each step requires the manual 


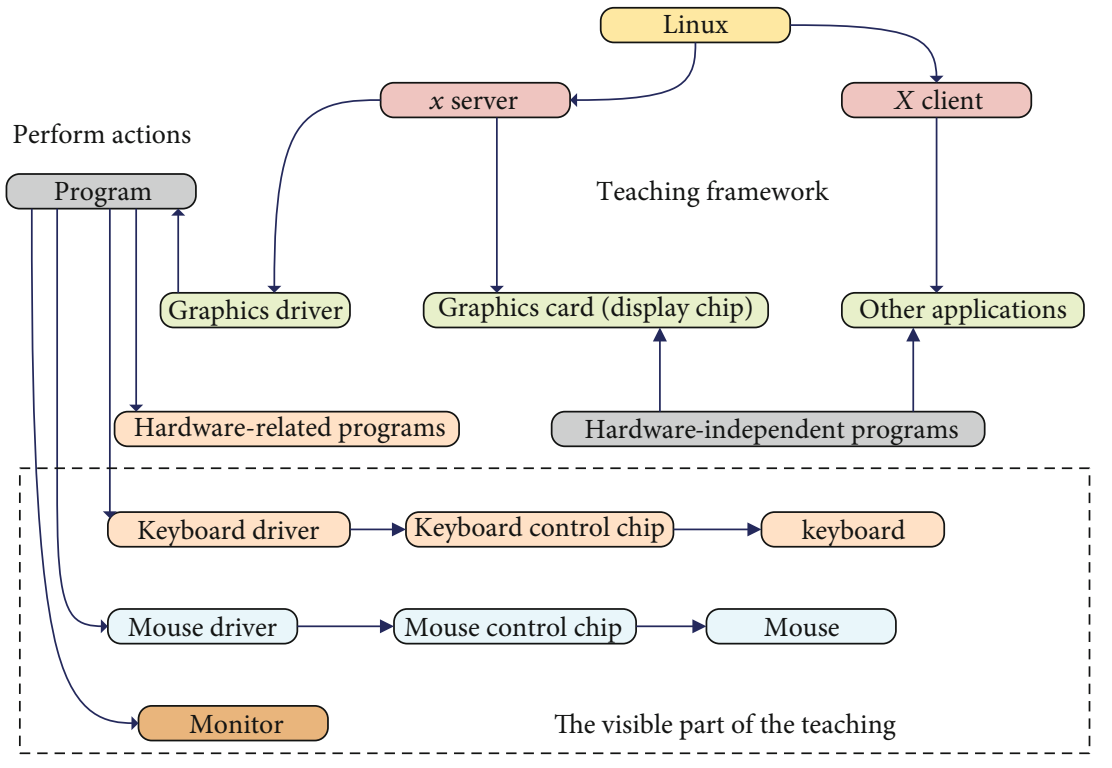

FIGURE 1: Intelligent teaching system physical education framework diagram.

establishment of patterns and rules, resulting in a heavy labor burden, and the completeness of rules and patterns needs to be verified, which limits its application scale [14]. Therefore, the research methods and application scale of this paper are obviously different. This paper makes a lot of empirical research on the statistical characteristics of different titles from the perspective of empirical software engineering. The research shows that there are statistically significant differences in the functional titles, bug repair titles, and security courseware repair titles. These experiences are the main basis for the definition and selection of features when doing feature engineering for code titles. However, the research methods and objectives of this paper are different from those of these studies [15].

\section{Results and Analysis}

3.1. IoT Cloud Platform Teaching Application Analysis. The Internet of Things is a network based on the Internet, traditional telecommunication networks, and other information carriers, so that all ordinary objects that can exercise independent functions can realize interconnection. This paper analyzes physical education teaching based on the Internet of Things platform. The cloud platform is a hardwarebased service that provides computing, network, and storage capabilities. The cloud platform allows developers to either run written programs in the "cloud" or use the services provided in the "cloud" or both. Cloud platforms are mainly divided into three categories: storage, computing, and comprehensive cloud platforms.

As shown in Figure 2, each source code title consists of a log message and code part. The former is the nature and behavior of the title described by natural language, while the latter specifies the modification (addition, deletion, and replacement) of the source code file. Open-source software develops and evolves in the form of merge, which can be divided into three categories: bug, function, and courseware repair. It is easy to get titles directly from the system kernel community; however, because of the randomness of title description information and title code, it is difficult to automatically classify (label) titles according to the title information. However, model training and updating need enough tagging titles, which require the method of continuous collection and tagging titles and the design and development of automatic tools.

As shown in Table 1, it is difficult and unreliable to collect security courseware titles from the Linux warehouse directly according to the title submission information. The courseware with CVE ID allocated in NVD physical education multimedia is confirmed and reliable. Therefore, this paper adopts the method of parsing information from NVD physical education multimedia and then tracing the GIT source code warehouse of the system kernel.

The Internet and multimedia technology particularly require students to have the ability to quickly screen and obtain information, creatively process and process information, and to take the ability of students to master and use information technology as a new basic ability that is as important as reading, writing, and numeracy. The teaching interface of the intelligent teaching system is shown in Figure 3. An accurate security courseware repair title can be obtained, which can be definitely labeled as courseware title and added into the annotation data set. Note that the extracted hash ID is invalid due to invalidation, error in reporting courseware, and other reasons, so it is necessary to check whether the hash ID is valid. Through the above method process, this paper can skillfully collect and label reliable missing report header data set for IoT cloud platform kernel and continuously update. Obviously, the courseware title annotation method can be easily extended to support other open-source projects.

As shown in Figure 4, the collection is not the title of security courseware repair, but the title of function enhancement or pure bug repair. These titles are labeled as nonvulns. 


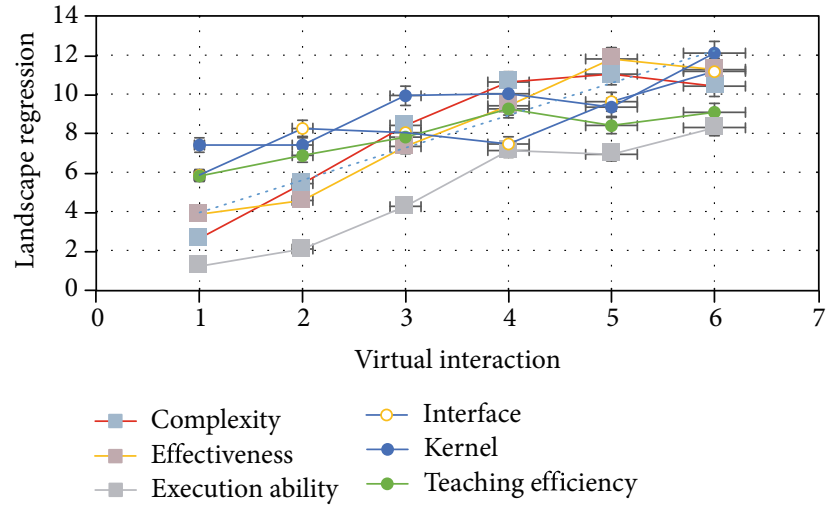

Figure 2: The effect of the specified source code file in physical education.

TABLE 1: Teaching multimedia analysis information effect.

\begin{tabular}{lccccc}
\hline Item & Complexity & Effectiveness & $\begin{array}{c}\text { Execution } \\
\text { ability }\end{array}$ & Interface & Kernel \\
\hline BMP & 7.96 & 8.48 & 9.51 & 5.75 & 8.28 \\
JPG & 5.26 & 8.58 & 7.11 & 2.93 & 5.72 \\
PNG & 2.64 & 7.41 & 8.1 & 2.95 & 6.56 \\
TIFF & 1.73 & 5.39 & 7.86 & 0.15 & 4.34 \\
GIF & 0.35 & 6.17 & 7.17 & -1.68 & 4.32 \\
\hline
\end{tabular}

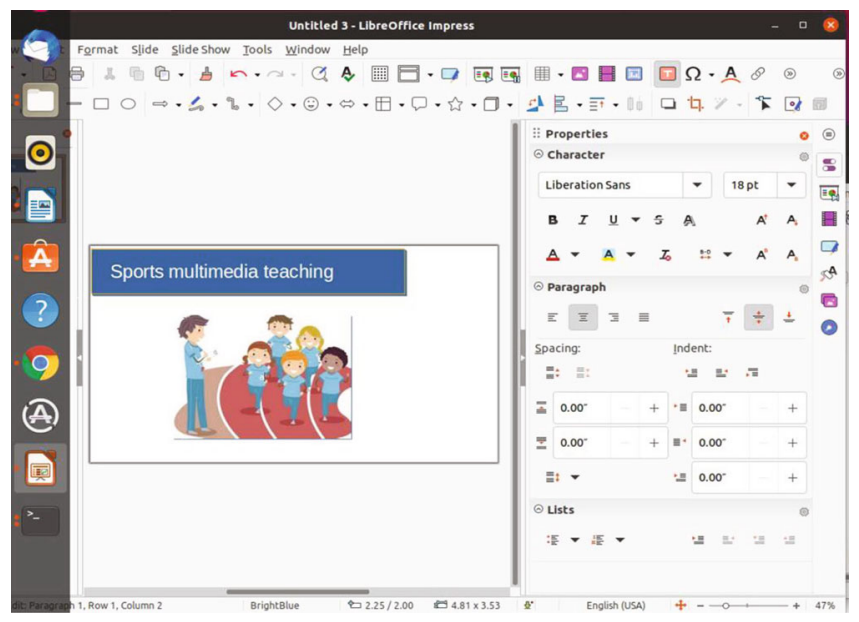

FIGURE 3: Multimedia physical education interface.

Collect clear bug repair titles: first, conduct preliminary screening through fix filter log message, then remove the titles with security-related sensitive keywords, then remove the titles clearly marked with security, and finally remove the titles with reference to security agencies. Collection function enhancement title is as follows: the keywords such as additional supporting, clean, clean up, and new feature appear in the log message, which is not included in the title of security courseware repair and bug repair.

As shown in Figure 5, the repair template abstracts a group of common changes between the same kind of errors and the corresponding title. Generating the title based on the

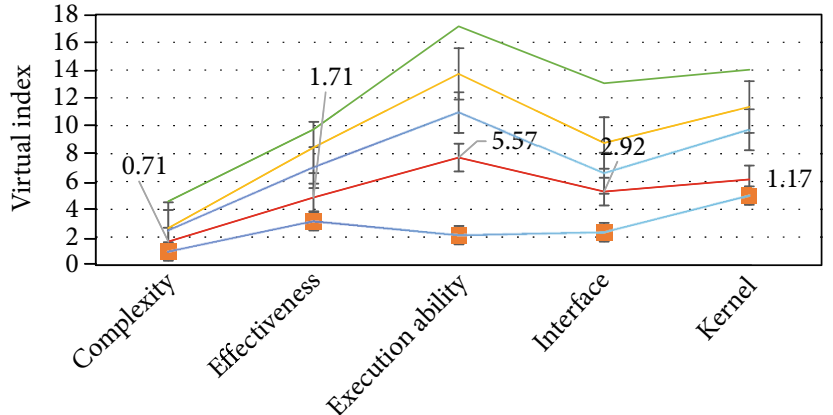

Experiments

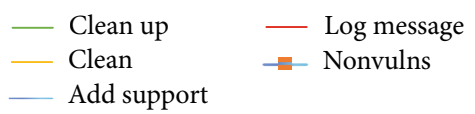

Figure 4: Title of safety courseware repair.

repair template is the process of specifying the selected abstract repair template by determining a variety of code elements. Therefore, the existing researches start from three aspects: extraction of a repair template, selection of repair template, and determination of diversified specific code elements.

As shown in Figure 6, when the intelligent teaching system kernel version is upgraded, some kernel interface service changes may be introduced due to the upgrade of the kernel version, which makes some kernel interface calls in the driver code no longer adapt to the updated kernel version. As a result, the original driver may produce inconsistent errors due to mismatching in the new kernel environment. In order to adapt to the frequently changing kernel versions and maintain the availability of existing devices in the new kernel environment, developers need to continuously modify the driver code and complete the driver migration. However, the kernel version change has a great impact on the driver. Due to the intelligent teaching system kernel version change, more than $35 \%$ of the adaptation modification lines are needed in the corresponding driver migration. A single change introduced in the intelligent teaching system kernel version may affect hundreds of code call points distributed in many different files.

As shown in Table 2, developers manually perform adaptation modification to complete this kind of migration work, which is a usually heavy workload and low efficiency and may introduce new errors. In view of the above problems, researchers have carried out research on the auxiliary adaptation of driver migration intermediate library and auxiliary information of driver migration. In order to improve the retrieval accuracy and efficiency of auxiliary examples, these works reduce the burden of driver transplant developers to a certain extent by providing auxiliary examples. However, developers need to further analyze the auxiliary examples and error codes and construct the adaptive title manually. The workload of manual repair is still large and the efficiency is low. Therefore, aiming at the inconsistent interface calls in driver migration scenarios, this paper recommends high-quality titles to reduce the workload of manual repair and improve repair efficiency. 

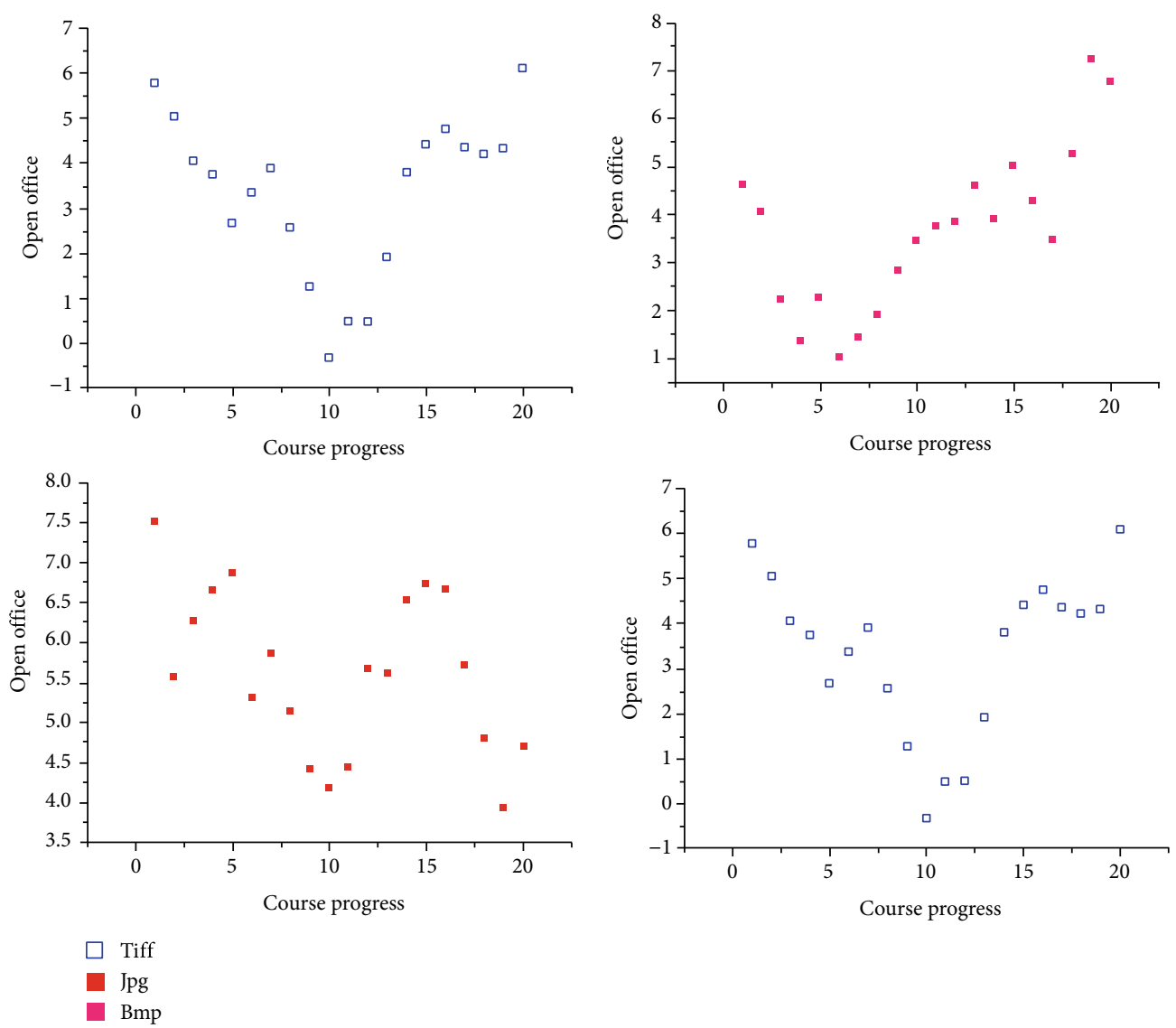

FigURE 5: Implied commonalities between similar errors and corresponding titles.

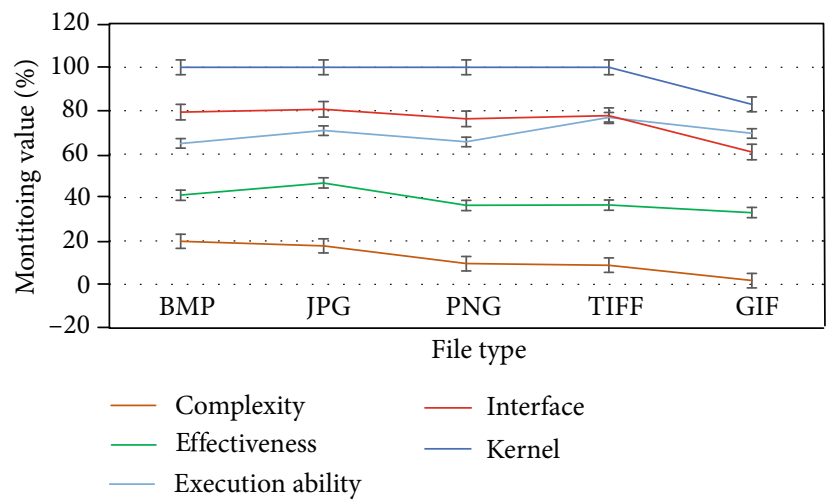

Figure 6: Incompatible with the kernel environment.

TABLe 2: Developers manually make adaptability.

\begin{tabular}{lccccc}
\hline Item & BMP & JPG & PNG & TIFF & GIF \\
\hline Complexity & -1.19 & 2.84 & 5.51 & -1.81 & 1.86 \\
Effectiveness & -3.76 & 2.71 & 3.28 & -2.87 & 2.01 \\
Execution ability & -2.76 & 2.92 & 2.38 & -2.82 & 1.1 \\
Interface & -3.39 & 2.83 & 1.95 & -2.07 & 2.07 \\
Kernel & -4.05 & 2.7 & 0.14 & -2.26 & -0.68 \\
\hline
\end{tabular}

3.2. Teaching Effect of Sports Courseware. As shown in Figure 7, compared with the auxiliary example, if there are high-quality titles to assist developers, the accuracy and efficiency of error repair will be greatly improved. Although both title recommendation and program auto repair need to generate titles for specific errors, they are different in error location and title quality judgment. Title recommendation is the process of generating titles and recommending appropriate titles for errors in the program, and finally, the quality of titles is determined by the developer manually.

As shown in Table 3, the main task of text is to generate and recommend titles for interface inconsistency errors in driver migration scenarios. Inconsistent interface calls in driver migration scenarios are actually a kind of specific error with characteristics. Different drivers reuse kernel interfaces through unified kernel interface services; therefore, a single point of kernel interface definition change caused by IoT cloud platform kernel version upgrade will lead to the same kind of inconsistent errors in different call points of the multiplex interface in multiple drivers.

As shown in Figure 8, the early research used a genetic algorithm to cross mutate reusable code fragments to generate titles, but this random mutation will produce a large number of meaningless titles. In order to improve the quality of the title, it is found that many errors have the same type. A group of repair instances of the same kind of errors actually contain repair templates. Therefore, for the same kind of 

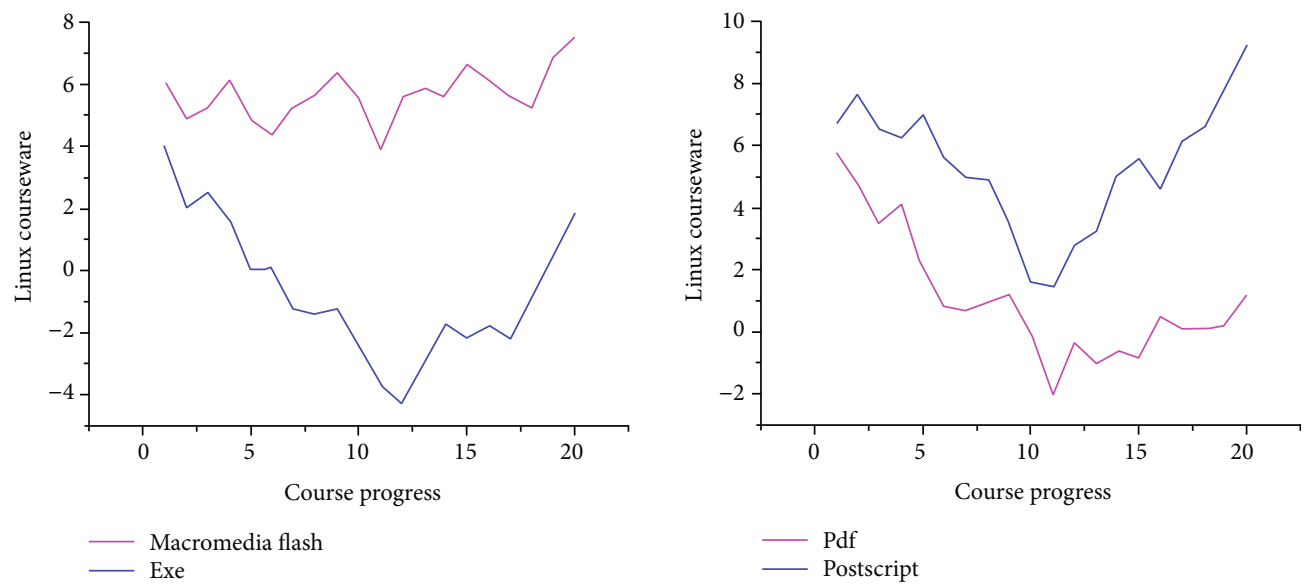

FIgURE 7: Error positioning and headline quality judgment.

TABLE 3: Interface call in a driver migration scenario.

\begin{tabular}{lccccc}
\hline Item & Effectiveness & Execution ability & Interface & Kernel & Teaching efficiency \\
\hline EXE & 7.72 & 10.97 & 5.24 & 7.62 & 3.75 \\
PDF & 8.6 & 10.57 & 4.89 & 6.49 & 2.87 \\
PostScript & 8.46 & 9.07 & 5 & 6.81 & 3.09 \\
HTML & 8.46 & 8.69 & 4.27 & 7.57 & 3.36 \\
Image & 8.22 & 7.03 & 4.62 & 6.52 & 2.32 \\
\hline
\end{tabular}

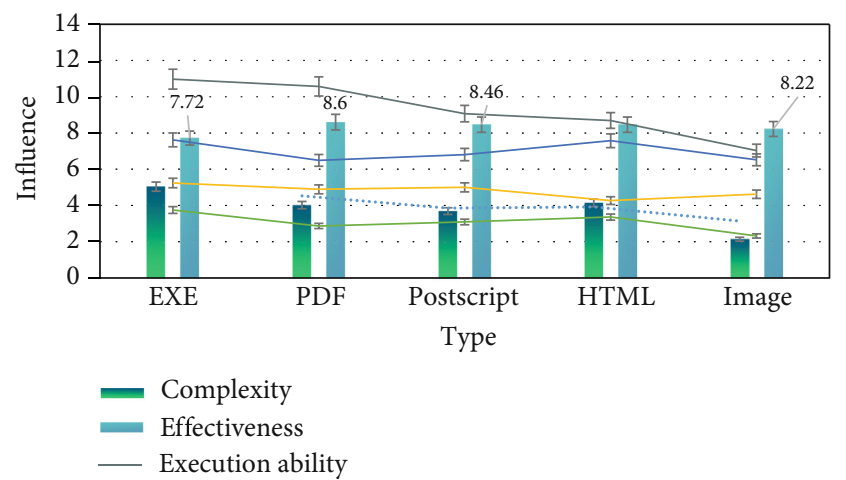

FIgURE 8: Cross-mutation of genetic algorithm to generate a title.

errors that have not been repaired, the title can be generated under the constraint and guidance of the repair template.

As shown in Figure 9, physical education teachers can improve the physical performance, physical quality, and health level of students by using systematic physical education. In physical education teaching, combining with students' professional knowledge will not only significantly improve the level of students' sports knowledge but also stimulate the interest and enthusiasm of sports students, and the professional knowledge of sports students will be applied and consolidated. Through the questionnaire, we can see that a large number of sports students like sports have formed a sense of active participation in sports and will independently arrange a reasonable time and frequency, scientific sports. The extensive development of physical education in sports colleges and universities can not only relieve the heavy learning pressure of sports students but also promote students' independent physical exercise and improve their comprehensive physical quality. About $60 \%$ of the sports students will arrange the frequency of the sport reasonably according to their own personal situation and carry on according to the plan for a long time, but 6 of them are doing unplanned sports. Although unplanned exercise can also achieve the purpose of adjusting emotions and releasing pressure, which is conducive to physical and mental health, compared with long-term reasonable exercise with a periodic schedule, it cannot achieve the purpose of significantly improving vital capacity, controlling weight, and preventing cardiovascular and cerebrovascular diseases.

As shown in Figure 10, the use of GIMP can greatly improve the learning efficiency in the physical education teaching of smart system. Compared with Windows and other systems, IoT cloud platform only retains the core components and can reflect the great advantage of system permissions in teaching. In terms of processing speed, the image processing time is $36 \%$ shorter than that of Windows, and there are more kinds of professional materials than Microsoft. Compared with Photoshop and 3D Max, the learning time of teachers using intelligent teaching system professional PE teaching software is shortened by more than $50 \%$, so as to improve the work efficiency of PE teachers.

\section{Discussion}

In order to rapidly improve the level of sports technology, climb the peak of world sports, and show the image of China, the state sports commission has adopted the mode 

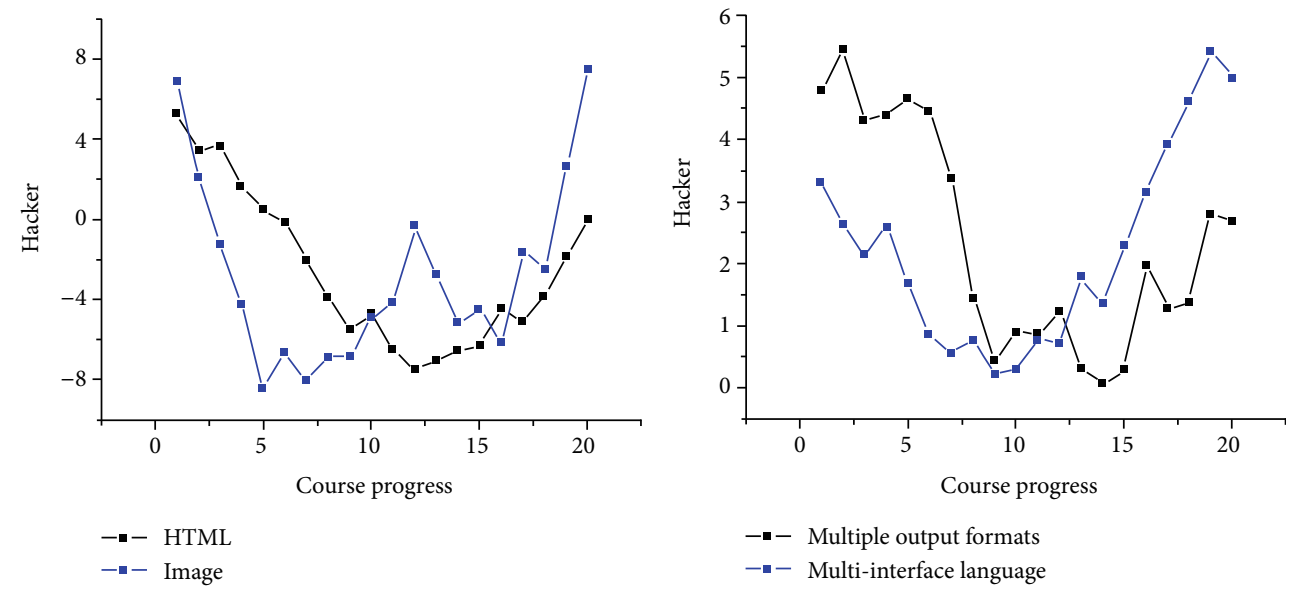

FIGURE 9: Expertise and knowledge level of the student's sports.
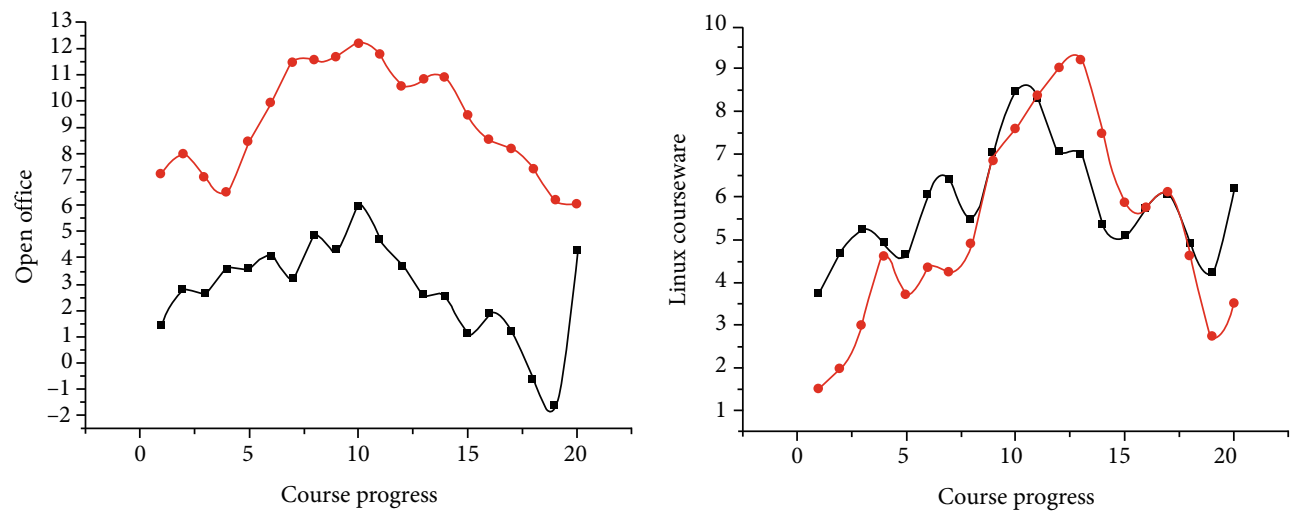

$$
\rightarrow \text { Png }
$$
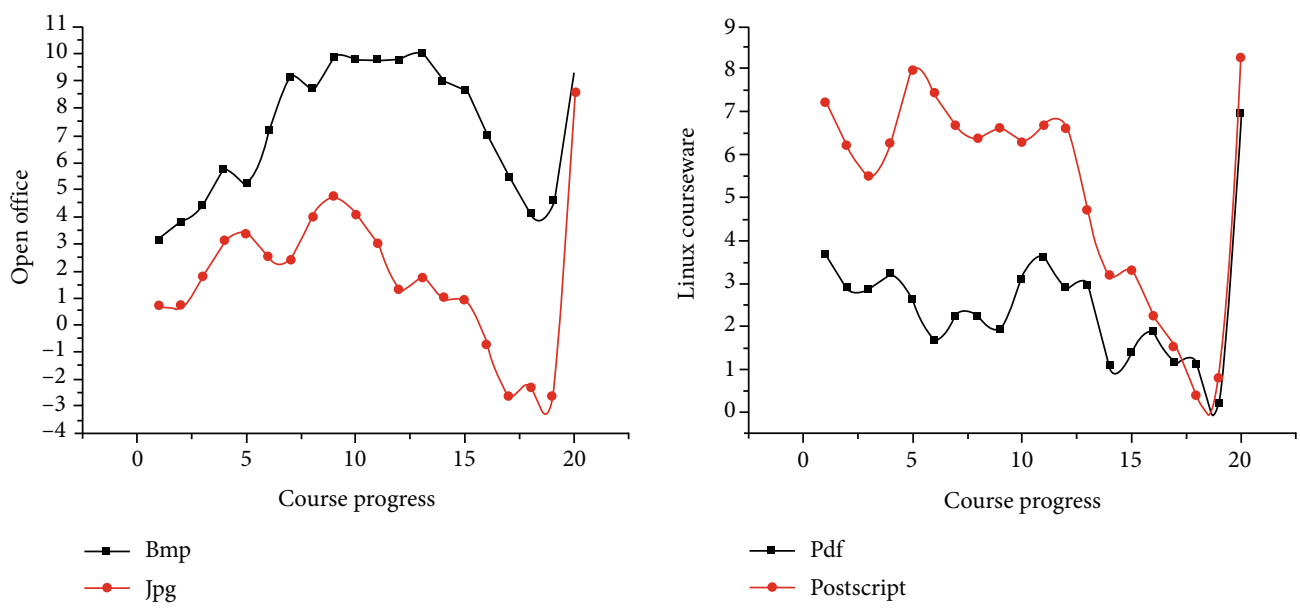

FIGURE 10: Learning efficiency of physical education in intelligent teaching system.

of "centralized accommodation, centralized training, and centralized management" to form an excellent sports team, taking the first step in the cultivation of competitive sports talents [16]. After years of practice and exploration, a three-level training network linking amateur and profes- sional training of China's competitive sports talents has been formed, which provides talent reserve and support for the rise of China's competitive sports [17]. Due to the fact that the competitive sports talents in the "three-level training network" are limited in the relatively closed sports system, 
to a certain extent, the overall level of cultural education of professional athletes in China is not high, and the channel of retirement placement is narrow, which affects the cultivation and selection of competitive sports reserve talents [18].

From the macro point of view, the mode of combination of sports and education lacks top-level design in the system and compatibility in the micro level, which leads to the lack of hierarchy in the implementation of a combination of sports and education, the lack of relevance in cohesion, the lack of directionality in development, and the lack of clarity in objectives, which are manifested in the weakness of the "three-level network" of competitive sports reserve talents and the weakening of the training function of competitive sports talents in sports colleges; it is difficult for ordinary colleges and universities to shoulder the responsibility of cultivating competitive sports talents alone. Competitive sports talents show a situation of shrinking scale and declining quality, which leads to the low cultural standard, low training requirements, and low export quality of competitive sports reserve talents [19]. In terms of sports training, there are some problems in colleges and universities, such as incomplete sports facilities, lack of high-level coaches, low ability of scientific research support, and less investment. In terms of cultural learning, the rigidity of curriculum, teaching content, teaching progress, and teaching methods makes it difficult to meet the learning needs of high-level competitive sports talents $[20,21]$. As a result of the insufficient supply of resources and misallocation of resources, it is difficult for competitive sports talents to meet the requirements of high-level competitive sports reserve talents in the level of sports technology, and there are few talents who can serve the country and participate in international competitions. In terms of disciplines and majors, although some excellent athletes have diplomas and academic qualifications, they do not have a competitive advantage in choosing jobs because they only meet the most basic standards required by the school for students. Whether competitive sports can maintain high-speed and healthy development, and whether the talent flow channel is smooth or not is an important test index. There are many problems in the flow channel of competitive sports talents in the mode of combination of sports and education [22]. It is difficult to recruit, select, and promote competitive sports reserve talents, and the shortage and overstock of talents coexist. There are many obstacles in the flow of talents among amateur sports schools, key sports schools, professional teams, traditional sports schools, primary and secondary schools, and colleges and universities. It is difficult for athletes to adapt to the education system and change their roles. It is difficult for highlevel sports team students to get equal selection and participate on behalf of the country, forming a horizontal blockage [23]. The closed-loop operation of the sports system undertakes the resettlement of retired athletes. Limited by their acceptance ability, the resettlement system of retired athletes, and the comprehensive quality and ability of athletes, it is difficult for retired athletes to choose jobs and obtain employment, resulting in the poor transformation of sports careers and careers [24]. The above problems have not been well solved for a long time, resulting in the quality and scale of training competitive talents with the combination of sports and education are far from expectations. Upgrading, transforming, and innovating the mode of combination of sports and education have become the inevitable demand and mission of the times for the development of competitive sports [25].

After many years of exploration, the reserve talent training system of "both reading and training, scientific training" in competitive sports and the school running mode of "teaching, scientific research, and training" in sports colleges and universities have achieved great success. A large number of outstanding young reserve talents and college athletes have come to the fore, many sports have achieved unprecedented excellent results, and a number of world champions and Olympic champions have been cultivated, which have attracted great attention at home and abroad. The successful practice of cultivating competitive sports reserve talents and high-level athletes in sports colleges and universities fully proves that sports colleges and universities in China can fully shoulder the important task of training excellent competitive reserve talents and high-level athletes. Unfortunately, there are some problems in the later stage, such as positioning swing, fuzzy role, poor mechanism, and so on, which push it to the edge of premature death. The issue of "opinions" and the proposal of the concept of "integration of sports and education" provide an opportunity for professional sports colleges to give full play to the advantages of cultivating high-level reserve talents of competitive sports [26]. "Multimedia courseware teaching" as a professional sports colleges to cultivate high-level competitive sports talent path in the new era reopened. The characteristics and advantages of "multimedia courseware teaching" are as follows: first, the early specialization of training. Sports colleges and universities are endowed with a complete system of education and training that connects middle schools and universities. They can carry out the hierarchical connection in the early specialized "scientific training" of sports technology for competitive sports talents, and at the same time, they can carry out cultural learning throughout the whole process, so as to realize the goal of educating people and talents in the "both reading and training". Second, professional advantages are highlighted. Sports colleges with sports as their professional characteristics have good supporting conditions, such as high-level coach team, high-quality scientific research team, first-class sports facilities, comprehensive sports discipline group, and environment and atmosphere for the growth of competitive sports talents, which provide necessary professional conditions for the cultivation of competitive sports talents. Third, the selection and success rate is high [27]. From sports talents to competitive sports reserve talents and then to the promotion of high-level competitive sports talents, competitive sports schools can enter provincial and municipal professional teams and then lead to the national team. The competitive sports talents who cannot achieve this promotion can realize the identity transformation from competitive sports talents to sports students in junior high school, senior high school, and university. This transformation is based on the successful practice of the "reading and training" style. Today, this path is used as the 
main channel for the cultivation of competitive sports reserve talents in China. The past experience and shortcomings are important references. In particular, it is necessary to implement the "reading" in the core of "reading and training" and the characteristics of multimedia courseware teaching, so that the cultivation of competitive sports reserve talents can be fully integrated into the national education system so that competitive sports reserve talents can really receive a systematic, complete, and high-quality education, and the "training" should be standardized at the same time; we should bring the cultivation of competitive sports reserve talents into the professional track of sports training and improve the success rate of talent cultivation on the basis of giving full play to the advantages of sports colleges in training, scientific research, and field.

\section{Conclusions}

Fully integrate and use these data as the basis for educational decision-making, which can improve the objectivity and scientificity of decision-making. The educational data mining cloud system is a software system that assists people to achieve these goals and has important research significance. The educational data mining cloud system spans multiple fields of education, data, and software and is still in its infancy. With the help of the intelligent teaching system, the teaching content of physical education in physical education colleges and universities is more closely related to the professional knowledge of physical education. Physical education teachers can combine the professional knowledge of physiology learned by students to teach, so as to improve students' enthusiasm for learning physical education courses, consolidate professional knowledge, and promote the development of students in all aspects. Compared with Photoshop and 3D Max, the learning time of teachers using intelligent teaching system professional PE teaching software is shortened by more than $50 \%$, so as to improve the work efficiency of PE teachers. Through the overall planning of the course, reasonable arrangement of teaching order, as far as possible to carry out a variety of forms of teaching, makes the classroom rich. The traditional method of extracting repair templates mainly uses similarity clustering to identify the repaired similar errors and abstracts the commonness of repair code changes to extract repair templates. But the intelligent system also has some problems, such as the system cannot achieve self-renewal and self-improvement, cannot achieve smooth communication with students, and the interaction is not enough.

\section{Data Availability}

No data were used to support this study.

\section{Conflicts of Interest}

The authors declare that there are no conflicts of interest regarding the publication of this article.

\section{References}

[1] A. Quach, Z. Wang, and Z. Qian, "Investigation of the 2016 Linux TCP stack vulnerability at scale," ACM SIGMETRICS Performance Evaluation Review, vol. 45, no. 1, pp. 8-8, 2017.

[2] M. Stuermer, G. Abu-Tayeh, and T. Myrach, "Digital sustainability: basic conditions for sustainable digital artifacts and their ecosystems," Sustainability Science, vol. 12, no. 2, pp. 247-262, 2017.

[3] P. Yuan, Y. Guo, L. Zhang, X. Chen, and M. Hong, "Building application-specific operating systems: a profile-guided approach," Science in China: Information Science (English Edition), vol. 61, no. 9, pp. 17-33, 2018.

[4] F. Block and A. Dewald, "Linux memory forensics: dissecting the user space process heap," Digital Investigation, vol. 22, no. 4, pp. S66-S75, 2017.

[5] K. Wang, A. Hussain, Z. Zuo, G. Xu, and A. Amiri Sani, "Graspan,” ACM SIGOPS Operating Systems Review, vol. 51, no. 2, pp. 389-404, 2017.

[6] M. Coudron and S. Secci, "An implementation of multipath TCP in ns3," Computer Networks, vol. 116, pp. 1-11, 2017.

[7] P. López and E. Baydal, "Teaching high-performance service in a cluster computing course," Journal of Parallel and Distributed Computing, vol. 117, no. 6, pp. 138-147, 2018.

[8] K. Belyaev and I. Ray, "Component-oriented access controlapplication servers meet tuple spaces for the masses," Future Generation Computer Systems, vol. 86, no. 9, pp. 726-739, 2018.

[9] P. Mishra and A. K. Somani, "Host managed contention avoidance storage solutions for big data," Journal of Big Data, vol. 4, no. 1, pp. 18-19, 2017.

[10] X. Xu, D. Cao, Y. Zhou, and J. Gao, "Application of neural network algorithm in fault diagnosis of mechanical intelligence," Mechanical Systems and Signal Processing, vol. 141, article 106625, 2020.

[11] K. Belyaev and I. Ray, "On the formalization, design, and implementation of component-oriented access control in lightweight virtualized server environments," Computers \& Security, vol. 71, no. 11, pp. 15-35, 2017.

[12] Y. Li, Y. Zuo, H. Song, and Z. Lv, "Deep learning in security of Internet of Things," IEEE Internet of Things Journal, 2021.

[13] L. K. Tsui and F. Garzon, "Carbon XS GUI: a graphical frontend for carbon XS," Journal of Applied Crystallography, vol. 50, no. 6, pp. 75-78, 2017.

[14] Y. Chen, X. Ye, and F. Huang, "A novel method and fast algorithm for MR image reconstruction with significantly undersampled data," Inverse Problems \& Imaging, vol. 4, no. 2, pp. 223-240, 2010.

[15] O. Hahm, E. Baccelli, H. Petersen, and N. Tsiftes, "Operating systems for low-end devices in the Internet of Things: a survey," IEEE Internet of Things Journal, vol. 3, no. 5, pp. 720$734,2016$.

[16] M. Gadelha, H. I. Ismail, and L. C. Cordeiro, "Handling loops in bounded model checking of C programs via k-induction," International Journal on Software Tools for Technology Transfer, vol. 19, no. 1, pp. 97-114, 2017.

[17] T. Yasuhiro, F. Takatomo, and N. Yasukazu, "DFAST: a flexible prokaryotic genome annotation pipeline for faster genome publication," Bioinformatics, vol. 58, no. 6, pp. 1037-1039, 2018. 
[18] L. Hahn and J. Moore, "Evaluation of a discrete dynamic systems approach for modeling the hierarchical relationship between genes, biochemistry, and disease susceptibility," Discrete and Continuous Dynamical Systems-Series B (DCDS-B), vol. 4, no. 1, pp. 275-287, 2004.

[19] A. Stein, R. Scheidler, and M. Jacobson, "Cryptographic protocols on real hyperelliptic curves," Advances in Mathematics of Communications, vol. 1, no. 2, pp. 197-221, 2017.

[20] J. Wang, L. Zhang, L. Duan, and R. X. Gao, “A new paradigm of cloud-based predictive maintenance for intelligent manufacturing," Journal of Intelligent Manufacturing, vol. 28, no. 5, pp. 1125-1137, 2017.

[21] N. Agarwal and T. F. Wenisch, "Thermostat," ACM SIGARCH Computer Architecture News, vol. 45, no. 1, pp. 631-644, 2017.

[22] S. Fujishige and S. Isotani, "New maximum flow algorithms by MA ORDERMGS and scaling," Journal of the Operations Research Society of Japan, vol. 46, no. 3, pp. 243-250, 2003.

[23] X. Ge, W. Cui, and T. Jaeger, "GRIFFIN," Acm Sigarch Computer Architecture News, vol. 45, no. 1, pp. 585-598, 2017.

[24] G. Cena, S. Scanzio, A. Valenzano, and C. Zunino, "Implementation and evaluation of the reference broadcast infrastructure synchronization protocol," IEEE Transactions on Industrial Informatics, vol. 11, no. 3, pp. 801-811, 2015.

[25] P. B. Leonard, E. B. Duffy, R. F. Baldwin, B. H. McRae, V. B. Shah, and T. K. Mohapatra, "gflow: software for modelling circuit theory-based connectivity at any scale," Methods in Ecology and Evolution, vol. 8, no. 4, pp. 519-526, 2017.

[26] J. Guo, F. Lin, X. Zhang, J. Zheng, and J. Zheng, "NetLand: quantitative modeling and visualization of Waddington's epigenetic landscape using probabilistic potential," Bioinformatics, vol. 33, no. 10, pp. 1583-1585, 2017.

[27] C. Zhou, M. Jin, Y. Liu, Z. Xie, and H. Liu, "Hybrid task priority-based motion control of a redundant free-floating space robot," Chinese Journal of Aeronautics, vol. 30, no. 6, pp. 2024-2033, 2017. 